\title{
Usage of Long Acting Reversible Contraceptive Methods among Women Who Want No More Children, In-Depth Analysis of the Ethiopian Demographic and Health Survey 2005
}

\author{
Seyfu Abebe Desta, Alemayehu Worku \\ School of Public Health, University of Gondar, Gondar, Ethiopia
}

Email address:

sababe@pih.org (S. A. Desta), seyfuabebe@gmail.com (S. A. Desta), alemayehuwy@yahoo.com (A. Worku)

To cite this article:

Seyfu Abebe Desta, Alemayehu Worku. Usage of Long Acting Reversible Contraceptive Methods among Women Who Want No More Children, In-Depth Analysis of the Ethiopian Demographic and Health Survey 2005. Science Journal of Public Health.

Vol. 5, No. 6, 2017, pp. 428-439. doi: 10.11648/j.sjph.20170506.14

Received: September 20, 2017; Accepted: September 28, 2017; Published: November 6, 2017

\begin{abstract}
Problem Statement: The Long Acting Reversible Contraceptive Methods were known to provide different benefits for the client and the health system when compared with the short acting methods. Despite these advantages, these methods are underutilized in the world in general and in Ethiopia in particular with a prevalence rate of $0.2 \%$. Detrmining the factors for usage of Long Acting Reversible Contraceptives among women who want no more children not only help to plan interventions and increase their utilization of these methods but also will have significant contribution to improve the health system provision due to the diversified benefit. The objective of this study was to figure out the main factor that determines the use of long acting reversible contraceptive methods among current users who want no more children in 2005 in Ethiopia. The in-depth analysis of this study was based on the Ethiopian Demography and Health survey conducted on 2005 G. C. Data pertaining to this study was taken from the survey and analyzed using SPSS 19. Multiple binary logistics regressions were used to find relationship between the independent and dependent variable using Complex survey analysis. After adjusting for demographic and reproductive health characteristics, highest education attained, ever had a terminated pregnancy, heard Family planning on radio, told about FP by health or FP worker and decision maker on use of contraception were found to be determinants for the use of Long Acting Reversible Contraceptives. The study has identified highest level of education attained, ever had a terminated pregnancy, heard FP on radio and decision maker on using contraception were found to be determinates. Approaches that address the mentioned factors will significantly enhance the use of the Long Acting Reversible Contraceptives in this subpopulation.
\end{abstract}

Keywords: Long Acting Contraceptives, Women, No More Children, Family Planning

\section{Introduction}

Immigration, death and fertility are the principal components of population dynamics which determine the size and structure of a population of a country. A fast growing population due to high fertility rate usually hampers the development of a country. Family planning (FP) is central to slowing fast growing population size and thus helps to contain the fertility of a country in suitable range. Parents use traditional or modern family planning methods either to space or limit child bearing. Most of the
Developing/sub-Saharan countries have unmet need for their family planning need and even among those that utilize modern methods the usage is skewed to specific methods mostly to the short acting injectable and pills [1]. A method that is very appropriate and comfortable during one stage of a women's life is often not a method she might want to use at a different time. Women who are unsatisfied by short acting methods but still wish to avoid pregnancy need alternative family planning choice. This includes women who are not ready to accept permanent methods but still need to limit the number of children they have.

The unmet need for family planning around the world in 
2009 was $11.2 \%$ and the specific figure to the eastern African region was reported to be $27.6 \%$. Besides the distribution of some type modern family planning methods have geographical differences even if the pill and the injectable are more prominent worldwide. The Eastern Europe countries like Uzbekistan (2006; 49.7\%,) and Kyrgyzstan (2005/2006, $32 \%$ ) were reported to have higher usage of the Intrauterine device (IUD). The prevalence of IUD usage in China, Cuba, Mongolia and Vietnam was above $40 \%$. Few African countries like Egypt and Tunisia have a prevalence of $36.1 \%$ and $27.8 \%$ in 2008 and 2006 respectively. These countries represent few part of the world and thus the prevalence of long acting reversible contraceptive methods; implants and IUD; (LARCS) is very low in most of the countries in this world [2].

The situation in Ethiopia is not different from the rest of the world regarding family planning issues and specifically concerning LARCs. The knowledge of married women about IUD and implants had increased from $10.2 \%$ and $13.2 \%$ in 2000 to $12.2 \%$ and $22.3 \%$ in 2005 , respectively. Despite an increase in knowledge the use of long term reversible contraceptive method had remained very low $(0.2 \%$ for both IUD and Implants) as of 2005. Furthermore around 5\% percent of non-user married women had wished to use implants in the future as noted in the 2000 Ethiopian Demographic and Health Survey (EDHS) but the need decreased to $1.7 \%$ in 2005 EDHS. Those who wished to use IUD had increased from $0.1 \%$ in 2000 to $0.3 \%$ 2005. The proportion of currently married women who preferred to limit the number of children in 2005 was $42 \%$ with, an increase of $30 \%$ from 2000 with proportionate increase in rural area higher than in urban areas. The desire to stop child bearing increases with the number of living children from nine percent among women with no children to 72 percent among women with 6 or more children. The proportion of women with unmet need for limiting has remained unchanged between the two consecutive EDHS surveys. [3, 4]

The usage of LARCs is very low in the world in general and in Ethiopia in particular. The under use is more pronounced for Implants than IUDs [2]. Attempts were made to know the related factors for low prevalence of modern contraception as a group and few to address individual methods and most ended up with factors related to the method, the client, the provider and health and logistics system. Fear of insertion, Fear of the design of the IUD, partner disappointment, rumors and myths as a baby will be born with IUD, the IUD can get lost in the body, embeds in the uterus, limited supply, time to discuss about the methods, provider discouragement, training needs, demand generation and promotion, cost etc were mentioned for low utilization of the method. [5, 6, 7]

Many studies were conducted to investigate those factors which determine the prevalence of modern contraceptive methods in group and at individual method level. To the best of the investigators knowledge none of the studies assess the usage of LARC methods among women who are using modern contraceptive to limit the number of children. LARC methods combine different features which make them the appropriate methods for this segment of women, like reversibility, effectiveness, cost-effectiveness, low need on client compliance and serving for long period of time. Moreover they save the precious time of health care providers and hence decrease the burden on the health system. This study which aimed to asses usage of LARCs among women who are currently using contraception to limit child bearing based on the 2005 EDHS, will not only uncover factors for low usage of the methods but will help in designing strategies which will help in increasing the usage of these methods with an ultimate goal of improved family planning service.

\section{Literature Review}

\subsection{Prevalence}

There have been differences with respect to contraceptive preference between regions and countries. This results in different contraceptive method mix or the share of use represented by each method. The Eastern Europe countries like Uzbekistan (2006 49.7\%), and Kyrgyzstan (2005/2006, $32 \%$ ) were reported to have higher usage of the Intrauterine device (IUD). The prevalence of IUD usage in China, Cuba, Mongolia and Vietnam was above 40\%. Few African countries like Egypt and Tunisia have a prevalence of $36.1 \%$ and $27.8 \%$ in 2008 and 2006 respectively. These countries represent few part of the world and thus the prevalence of long acting reversible contraceptive methods (implants and IUD) is very low in most of the countries in this world. [2, 6, 8] In almost all countries of the world the prevalence of implant is less than $10 \%$. [2]

The prevalence of LARCs is lowest in sub-Saharan Africa with rate below $2 \%$ among women of reproductive age while the use of SARCs is very popular in this region. Even if $21.8 \%$ of married women of reproductive age living in sub-Saharan Africa reported of any contraceptive method use for the period of 2000-2005, the proportion of all use accounts for by IUD and pill decreased over the period of 26 years (1980-2005) while the share of injectable rose in the area and also worldwide. The share of injectable in sub-Saharan Africa grew from $8 \%$ to $26 \%$ over this period showing dramatic and consistent increase. $[2,9,10]$ In a survey to determine unintended pregnancy in sub-Saharan Africa: Magnitude of the problem and potential role of contraceptive implants has substantiated that the most prevalent method was combined oral contraceptive, 37\%, while IUD and injectable accounted for $8 \%$ and implant $0 \%$ $[1,2,9,11,12]$.

\subsection{Effectiveness and Cost Effectiveness}

Contraceptive effectiveness in preventing unintended pregnancy is influenced by efficacy, compliance, continuation over time, fecundity and coital frequency/timing.

Within the first year of perfect use of pill and 
Depo-Provera.0.3\% of women experience unintended pregnancies while it is $0.6 \%$ for IUD and $0.05 \%$ for Norplant When it comes to typical use, percentage of women who experience unintended pregnancy while using Implants and IUD remains almost similar but $8 \%$ pill users and $3 \%$ injectable users experience method failure. In general, annual pregnancy rate during implant, copper IUD and sterilization use were less than one per 100 women-years. $[13,14]$

If just one percent of current oral contraceptive and DMPA users in sub-Saharan Africa wanted contraceptive protection for five years and if they have an opportunity to choose and use an implant, then 93,000 pregnancies could be averted [1]. Thus the long term reversible contraceptives methods have the advantage of being reversible and highly effective.

These long acting reversible methods are not only effective but also they are cost effective. LARC methods are pointed to be cost effective than short term methods despite the high initial cost. Implant was the most effective and IUD was the cheapest option. [15]. The cost of Implanon per pregnancy avoided was extremely small when compared with Oral contraceptive pills. [4, 16]

\subsection{Choice of Method}

Different studies had tried to dig out the reason why clients choose a specific contraception method and come up with different factors. Being in relationship, balanced information, effectiveness to prevent pregnancy, side effects, ease of use, sexually transmitted infections (STI) prevention, previous unintended pregnancy, previous problem with other method, long term benefit, practitioner advice, education, Income, number of pregnancies, the age of the client etc were mentioned as reasons for choosing the current method they are using. [12, 17, 18, 19, 20, 21]. In a study to identify factors associated with contraceptive choice and inconsistent method use, in USA in 2004, where 38\% were using oral contraceptive while 5\% used IUD, pill use was less common among women in relationship for more than 4 years, more common among women who had an unintended pregnancy. [17]

Contraceptive choice depends on how well it prevents pregnancy $(56 \%$ in india, $51 \%$ in Jamaica), how few side effects it has $(26 \%$ in india, $11 \%$ in Jamaica), ease of use (10\% in India) and STI protection (19\%) in Jamaica. [12] In one study, the age range of women with ENT implant was 16-48 yrs. (median age 29, IQR 14-75), and ease of use, previous problems with other methods, long term benefit and practitioner advice were the main reasons to opt this method in descending order. In this similar study, an age range of 20-53 (median 34.5) women had had LNG IUS coil inserted, justifying for choosing the method as its ease and convenience of use, "lighter period", few side effects and because it was long term and reversible. [18] In a study comparing satisfaction with Norplant and tubal ligation, women who chosen Norplant were more educated, had a high family income, younger by an average of 5 years and had fewer pregnancies. In this study, $71 \%$ chosen Norplant for spacing and $50 \%$ users indicated desire for no more children. [19]. In a study for evaluating contraceptives choice through the method mix approach, given balanced information, $61 \%$ accepted IUD, 5.5\% Oral contraceptive and $4.5 \%$ Norplant. [20] Ease of use and effectiveness of Norplant were the two most features liked by clients in Nigeria and Ghana. [21].

The main reason why women chose long acting reversible contraceptive methods was because it was recommended by the provider followed by previous method unsuitable. At six month, more than $80 \%$ of respondents were still using the long term methods [21]. The most important reason for current contraceptive choice was effectiveness, followed by cost, potential side effects profile, ability to become pregnant in the future recommended, by provider and ease of use. Ease of use ranked within the top three most important reasons for current choice as patient education level increased from high school/GED or less to college graduates. [11]

\subsection{LARCs Use}

Few studies tried to figure out the use of LARCs and the related factors including barriers for low prevalence of these specific methods. LARC use among US women using contraceptive increased from $2.4 \%$ in 2002 to $5.6 \%$ in 2006-2008, the largest being among youngest and oldest group, and those in highest income group. The odds of LARC use differed significantly by the number of additional births expected, associated with total number live births, with a visit to a clinic for FP service in the past year and having stopped using a non-LARC method because of dissatisfaction. In this study after adjusting for demographic and sexual and reproductive health characteristics, in comparison with non-long acting reversible hormonal method users, women older than 35, married women, women who had ever experienced an unwanted pregnancy and women who had ever stopped using a non-long acting reversible hormonal method because of dissatisfaction were all more likely to be LARC users. [22]

Among a recent intervention to increase the uptake of LARCs in Zambia through dedicated midwifes showed that the program was able to provide LARC for 33,609 women among which two-third chose and the remaining selected IUD. Implant acceptors were younger and have fewer children than typical women in Zambia. IUD users were more likely to be limiters while about $75 \%$ of implant users desired additional children in the future. [23]

In a South African study to assess the knowledge, attitude and practice of IUD users, only a quarter of the participants had heard of IUD and less than $2 \%$ had used the method. The IUD might move to another place in the body, it might harm the womb; unspecified side effects were some of the misconception of clients heard about IUD. Being used for long time, effective, quick return of fertility were the advantages mentioned by clients after being given information. In this study few providers suggested IUD to suggest clients to seeking contraception and women unawareness was mentioned for the low copper use. [24]

In a study to find out trends and Determinant of 
Contraceptive Method Choice in Kenya, the probability of using long term methods is higher in urban than rural areas. A community exposed to family planning media messages on the radio is generally associated with a higher probability of use of long term and permanent methods and a low use of short term methods. The most popular method among older limiters $(>35)$ is sterilization, while the pill and injectable are the most popular methods among limiters younger than 35 . [9]

Limited researches have pointed why the use of LARCS mostly for IUDs is low and the factors are related to the client, the provider and the method itself. The most common reasons for not using IUD were fear of insertion (33.7\%) and fear of partner disappointment as pointed out in a study to explore acceptability of IUD among women in El Salvador. Over all, the participants in this study had a poor comprehension of IUD efficacy and adverse effect. [6]

In a qualitative study conducted in El Salvador, the most common reason for negative impression about IUD among clients was fear which was generally based on rumors and myths. Based on providers view about the rumors and myths, cancer, a baby will be born with the IUD in its body, an IUD can get lost in a women's body and IUD becomes embedded in the uterus were mentioned. The most common rumor that providers reinforced was that the IUD can move around in the body while visited by simulated clients. A limited supply of IUD and time to discuss about the IUD were mentioned as reasons for low level of IUD use by the providers. [7]

Training, cost of the IUD, demand generation and promotion, logistic and supplies were mentioned as barriers for Long Acting Reversible Contraceptives use. [5].

\subsection{Reason for Discontinuation}

Several methods related and other factors were identified for contraceptive method discontinuation. The relative continuation rate was found to be more in LARC users than short term methods. Percentage of women who continued use at one year was $68 \%$ for pill, $56 \%$ for DMPA, $78 \%$ for copper $\mathrm{T}$ and $84 \%$ for Norplant. [13] The three dominant reasons for discontinuation in six developing countries were health concerns including side effects, method failure and desire for a child with IUD discontinuation rate is lower than other methods. [25] Inconvenience to use for both the pills and injectable were mentioned as a reason for discontinuation in the 2005 EDHS. [4]

Side effects were given as a reason for not initiating contraception and as a main reason for either switching or discontinuing hormonal contraception all together. Among the stated side effects of implants, excessive and or constant bleeding was the commonest, most disliked side effect and which lead to most of the removal of the method while abdominal pain, expulsion and vaginal bleeding and discharge were attached with IUD for discontinuation and switching. [4, 18, 21, 26, 27, 28]

In most studies, it was possible to demonstrate that the long term reversible methods, Norplant and IUD had annual continuation rate above 90 per 100 women entering each year relative to the continuation rate for OCP (oral contraceptive pills) and injectable. Norplant continuation rate at one year was $90 \%$ in the Zimbabwe study and one other study identified the discontinuation rate for IUD to be $13.3 \%$. [14, $19,29]$. In a study investigating factors for affecting continuation rate of DMPA, where $2 / 3$ of the participants desired no more children, and more than $40 \%$ had 4 or more children, the 3, 6, 9 and 12 month continuation rates were $68 \%, 67 \% 55 \%$ and $51 \%$, respectively. [30] Unadjusted median 12-month discontinuation rates, from median value of five sub-Saharan countries for oral contraceptive and injectable were 46.2 and 32.0 respectively. [1]

Which method a women uses has important implication for how successful she will be in preventing pregnancy. Moreover a method that is very appropriate and comfortable during one stage of her life is often not a method she might want to use at a different time. Women who are unsatisfied by short acting methods but still wish to avoid pregnancy need alternative family planning choice. This includes women who are not ready to accept permanent methods but still need to limit the number of children they have. Most of the studies did not address these segment of women population for whom LARCs are the preferred method. This study, therefore, will try to add knowledge about contraceptive usage to the existing pool by focusing on LARCs and women who are currently married and contraceptive method users but who wished to stop child bearing.

\section{Objective}

\subsection{General Objective}

To determine factors that explain the usage of Long Acting reversible contraceptives among women who wanted to limit the number of children based on EDHS 2005.

\subsection{Specific Objective}

To figure out the main factors that determine the use of long acting reversible contraceptive methods among current users who want no more children.

To determine what are the attributes for the wide range disparity between long term and short term family planning methods among current users who want no more children

\section{Methods}

\subsection{Study Setting}

The in-depth analysis of this study was based on the Ethiopian Demography and Health survey conducted on 2005 G. C. Ethiopia, situated in the horn of Africa, is a populous country with a $73,918,505$ population size having diversified culture and language. At present Ethiopia is administratively structured into nine regional states, namely, Tigray, Affar, Amhara, Oromiya, Somali, Benishangul-Gumuz, Southern Nations, Nationalities and 
Peoples, Gambela and Harari regional states and two city administrations, that is, Addis Ababa and Dire Dawa Administration Council. Agriculture remains the main back bone of the economy in the country which employs $84 \%$ of the population that are living in the rural areas. Being dependent on traditional way of farming, the agriculture sector did not help the economic development of the country. Due to this poverty is the main problem of the country which is reflected on poor health condition mainly the communicable infectious diseases like HIV/AIDS, tuberculosis, Malaria etc. Moreover the country is known to have high fertility rates and thus remarkable population growth rate $(2.6 \%)$, high maternal and child mortality rate [31].

\section{Study design:}

The study design used was based on analysis of the cross-sectionally collected data of EDHS 2005 limited to Women who are currently using modern contraception for child limiting with specific focus on long acting reversible contraceptive methods.

\subsection{Study Population}

The 2005 EDHS sample was designed to provide estimates for the health and demographic variables of interest for the following domains: Ethiopia as whole; urban and rural areas of Ethiopia (each as a separate domain); and 11 geographic areas (9 regions and 2 city administrations), namely: Tigray; Affar; Amhara; Oromiya; Somali; Benishangul-Gumuz; Southern Nations, Nationalities and Peoples (SNNP); Gambela; Harari; Addis Ababa and Dire Dawa. All women aged between 15 and 45 which participated in the EDHS of 2005 are the source population from which mothers who are using contraception for child limiting will be the actual study subjects.

\subsection{Sample Size}

In the 2005 EDHS a representative sample of approximately 14,500 households from 540(145 urban and 395 rural) clusters were selected. In the first stage, 540 clusters were selected from the list of enumeration areas (EA) from the 1994 Population and Housing Census sample frame. In the second stage 14,500 houses were systematically selected from the cluster to participate in the census. Therefore, the data for EDHS 2005 were collected from nationally-representative probability samples of adult women and men in the reproductive age groups 15-49 and 15-59 with a sample size of 14,070 women and 6,033 men, respectively. For this specific study 690 (weighted) numbers of currently married women who want no more children and use modern contraceptive method were analyzed for usage of LARC methods.

\subsection{Sampling Procedures}

The 2005 EDHS samples were collected using a two-stage cluster sampling method. In the first stage, clusters were selected from the list of census enumeration areas using the
1994 Population and Housing Census sample frame. In the second stage, a complete listing of households in each selected cluster was carried out and households were selected systematically for participation in the surveys.

Data collection procedures:

The source of data for this study was EDHS 2005. Data pertaining to this specific study was collected using structured questioner which was developed in English language commented by different stakeholders and finalized. Latter the questioner was translated to three languages namely Amharic, Oromiffa and Tigrigna. The data collection tool had House hold questioner, Women`s Questioner and Men`s questioner.

The variable of outcome of interest is the use of long term reversible contraceptive methods among women who want no more children while the independent determinate used for this analysis were socio-demographic and sexual and reproductive health variables. Age, region, type of place of residence, highest education level, religion, ethnicity, current marital status, partners educational level, respondent currently working, were among the social-demographic variables used while ever had terminated pregnancy, wanted last child, heard family planning on radio last month, heard family planning on TV last month, visited by family planning worker in the last 12 months, at health facility told about family planning methods, told about side effect by family planning worker, told about other family planning method by family planning or health worker, husbands desire for children, decision maker for using contraception were the sexual and reproductive variables analyzed for their effect on LARCS use.

The Women's Questionnaire was used to collect information from all women age 15-49 years and covered areas like Household and respondent characteristics, Fertility levels and preferences, Knowledge and use of family planning and more issues on maternal and child health.

A pre-test was conducted from 11-25 February 2005 in four selected sites. The areas selected for the pretest were urban Addis Ababa and both urban and rural parts of Mekele, Ambo and Debre Birhan areas. Then the data was collected from April 27 to August 30, 2005 in the selected households across the country by 30 data collection teams each composed of four female and two male interviewers, one female editor and one male supervisor. The data collectors were trained, given examination beforehand and 240 were qualified and selected to participate in the interview.

Operational definitions:

1. Long acting reversible contraceptive methods: - are modern methods of contraception which provide long term protection from getting pregnant for at least three years which include Implants and intrauterine devices.

2. Short acting hormonal contraceptive methods:- are modern methods of contraception which provide short term protection not for more than three months and restricted to oral contraceptive pills and the injectable

3. LARCs users:- are those women who are using implants and IUDs for limiting the number of children 
4. LARCs non users:-are those women who are not using LARCs but rather chosen to use short acting hormonal contraceptives
5. Method:-For this thesis method is a contraceptive used to prevent pregnancy and could be either LARCs or SARCS
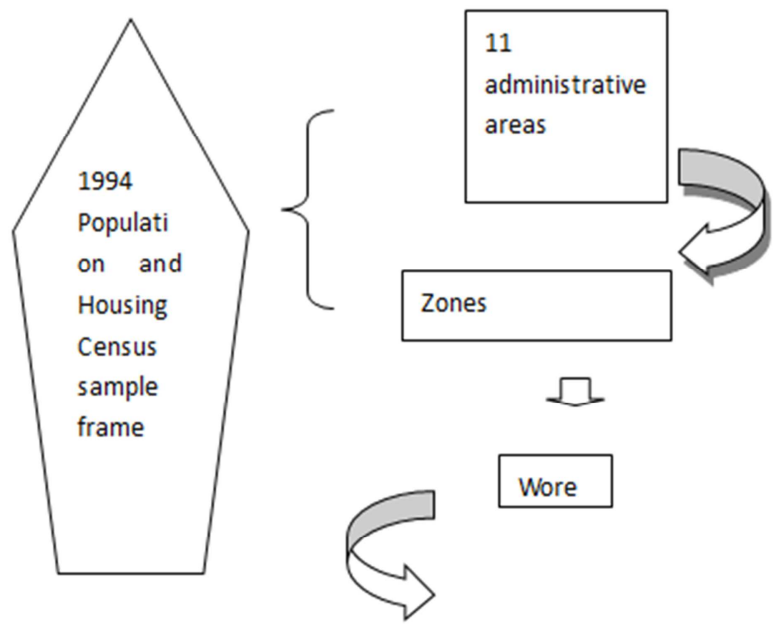

Census

enumeration areas

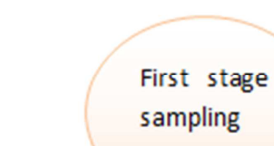

Clusters
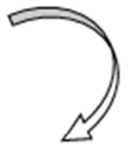

Households

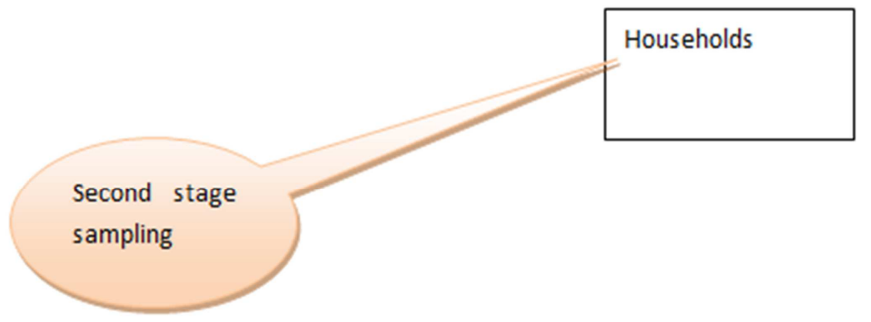

Figure 1. Schematic presentation of sampling procedure of the 2005 EDHS.

Data Management:

Each of the questionnaires was keyed twice by two separate data entry clerks. Consistency checks were made and entry errors were manually checked by going back to the questionnaires. A secondary editing program was then run on the data to indicate questions that showed inconsistency and these were also corrected by secondary editors.

Data Analysis procedures:

The analysis was restricted to current users of contraception who desired to limit the number of children they want. The data used for this analysis was the response collected from the women questioner. The desire for more children variable was recoded to assume responses "desire for more children" and "want no more" to separate the target group of interest. Likewise recoding was done to generate a variable which can accommodate the dependent variable and other independent variables were also recoded to suit for analysis after selecting the factors to be investigated. Complex sample cross tabulation and logistics analysis were used to describe frequencies and look at association to account for the two stage cluster sample design of EDHS 2005 , respectively. Selected individual independent variables were entered in binary logistic regression to see association through unadjusted odds ratio and those who were found to be determinant in the bivariate analysis were analyzed all together to see association after adjusting for individual determinants. In all analysis $95 \%$ CI was calculated for the odds ratio.

\subsection{Ethical Consideration}

Ethical clearance was obtained from University of Gondar and Macro International Inc. All ethical concerns and procedures were addressed in the EDHS 2005 and study subjects were informed and consented for participation.

Dissemination of results:

The study findings will be communicated to the Central statistics agency, Macro International Inc., Federal Ministry of Health and stake holders which are involved in the 
Reproductive Health and family planning services through journals.

\section{Result}

A total of 690 women who want no more children and using either short term or long term reversible contraception were selected for analysis. The current mean age of the respondent was 32.62 (CI: 32.324 to 33.01 ) and most of the respondents fall in the age group of 35-39 accounting for $171(20.9 \%)$ followed by age group of $25-29$ which takes $147(20.9 \%)$. Oromiya was represented by $248(35.5 \%)$ followed by Amhara region accounting for 216(31.4\%) cases study subjects; Harari and Gambela were the least represented with only $3(0.4 \%)$ cases. Higher proportion $71 . \%(491)$ of the study subjects were residing in the rural area and $61.8 \%(427)$ had no education at all. Majority of the study subjects were from orthodox religion representing $62 \%$ (428) and most of them 44.3\%(305) were from Amara Ethnic group. More than half, $63.8 \%(450)$ of the study subjects were not working and about $18 \%(128)$ who responded as having an occupation, they were agriculture employee.

As to the total number of children ever born; from 683 study subjects, $369(54.1 \%)$ of the women gave birth to $1-5$ children while $286(41.9 \%)$ gave birth to $6-10$ children and $27(4 \%)$ never. By the time of the survey, $452(65.4 \%)$ of the subjects had 1 to 5 living children while $239(34.6 \% 4)$ of the mothers had between 6 to 11 living children.

More than $640(92 \%)$ of the cases were married by the time of interview and $397(57.8 \%)$ and $279(40.6 \%)$ of them were between the age of 7 to 15 and 16 to 25 at their first marriage, respectively. Among all the study subjects, 614(89\%) reported to have no terminated pregnancy and from a total of 522 mothers, 243(46.7\%) wanted their last pregnancy, $199(38 \%)$ did not want at all and $80(15 \%)$ wanted it to happen later.

Almost $90 \%$ of the respondents ' husband/partner did know that the respondent was using the mentioned contraceptives and about $40 \%$ of the partners want the same number of children as the respondent.

Most of the respondents were not exposed to FP information through mass media; 401(58\%), 589(85.4\%) and $637(92.2 \%)$ were responded as they did not hear or read information on FP thorough radio, Television or newspaper, respectively. Moreover, nearly 86\% (N=597) women were not visited by FP worker and from those who visited health facility, less than $50 \%(\mathrm{~N}=162)$ were told about contraception More than $90 \%$ of the study subjects $(\mathrm{N}=404)$ were not told about side effect of contraceptives neither by FP nor health worker. Among those women who did not have desire for more children only $23(3.4 \%)$ were using LARCS while $667(96.6 \%)$ were using SARCS. From those women who want no more children, 277 discontinued their last contraceptive method and from those who were currently using LARCs $(\mathrm{N}=9)$ in this group $6(61 \%), 2(18.1 \%)$ and $1(10.4 \%)$ women discontinued injection, pill and IUD as their last method, respectively. Health concern and wanted more effective method were mention as a reason for last method discontinuation accounting for about 3(38\%) and $4(41 \%)$, respectively in the LARCs group.

Among the socio-demographic factors; region, residence, education, ethnicity: and among the reproductive health characteristics; ever had terminated pregnancy, heard family planning on radio and TV, told about side effect, told about other FP methods by health or FP worker, decision maker for using contraception was found to be significantly associated with the use of LARCs as a method of contraception.

Highest level of education attainment has a positive influence on the use of LARCs as a method of contraception. The odds of using LARCS are $42.725(95 \%$ CI: $8.118-224.855)$ times higher in women with higher education than no education. Being from Hadiya ethnic group favors the use of LARCs when compared to Amhara ethnic group with odds ratio of $34(95 \%$ CI: 2.236-517.136). The odds of using LARCS is $0.152(95 \% \mathrm{CI}: 0.064-0.385)$ times higher for those women who reside in rural area than urban area. (table 1)

Table 1. Percentage of Current Contraceptive Method Users; and Unadjusted Odds Ratios and 95\% Confidence Intervals for Current Use of Long-Acting Reversible Methods by Selected Socio-Demographic Characteristics, Ethiopia, 2005.

\begin{tabular}{|c|c|c|c|c|c|}
\hline \multirow{3}{*}{$\begin{array}{l}\text { Socio-demographic characteristics } \\
\text { Age }\end{array}$} & \multicolumn{4}{|c|}{ Method use } & \multirow{3}{*}{ Unadjusted OR (95\%) } \\
\hline & \multicolumn{2}{|c|}{ SARCS } & \multicolumn{2}{|c|}{ LARCS } & \\
\hline & No. & $\%$ & No. & $\%$ & \\
\hline $15-29^{\mathrm{a}}$ & 139 & 20.9 & 3 & 13.2 & 1 \\
\hline $30-34$ & 127 & 19 & 4 & 16.6 & $1.243(0.256-6.041)$ \\
\hline $35-39$ & 159 & 23.9 & 8 & 35.4 & $2.218(0.509-8.894)$ \\
\hline $40-44$ & 108 & 16.3 & 5 & 21.9 & $1.9(0.408-8.842)$ \\
\hline $45-49$ & 56 & 8.4 & 3 & 12.9 & $2.234(0.344-14.52)$ \\
\hline \multicolumn{6}{|l|}{ Education } \\
\hline No Education & 416 & 62.4 & 11 & 45.1 & 1 \\
\hline Primary & 164 & 24.6 & 7 & 29.2 & $1.263(0.399-3.999)$ \\
\hline Secondary & 79 & 11.8 & 3 & 13.5 & $1.233(0.272-5.588)$ \\
\hline Higher & 7 & 1.2 & 3 & 12.2 & $42.725^{* *}(8.118-224.855)$ \\
\hline \multicolumn{6}{|l|}{ Ethnicity } \\
\hline Amhara & 293 & 44 & 12 & 51.8 & 1 \\
\hline Gurage & 14 & 2.2 & 2 & 7.1 & $2.612(0.462-14.774)$ \\
\hline
\end{tabular}




\begin{tabular}{|c|c|c|c|c|c|}
\hline \multirow{3}{*}{$\begin{array}{l}\text { Socio-demographic characteristics } \\
\text { Age }\end{array}$} & \multicolumn{4}{|c|}{ Method use } & \multirow{3}{*}{ Unadjusted OR (95\%) } \\
\hline & \multicolumn{2}{|c|}{ SARCS } & \multicolumn{2}{|c|}{ LARCS } & \\
\hline & No. & $\%$ & No. & $\%$ & \\
\hline Tigre & 45 & 6.8 & 2 & 6 & $0.796(0.288-3.280)$ \\
\hline Others & 107 & 16.1 & 3 & 11.6 & $1.086(0.195-3.247)$ \\
\hline \multicolumn{6}{|l|}{ Religion } \\
\hline Orthodox & 413 & 62.0 & 15 & 63.5 & 1 \\
\hline Catholic & 9 & 1.4 & 0 & 0 & $0.347(0.118-1.017)$ \\
\hline Protestant & 129 & 19.4 & 3 & 13.9 & $0.7301(0.193-2.759)$ \\
\hline Muslim & 102 & 15.3 & 5 & 22.6 & $1.556(0.516-4.757)$ \\
\hline Traditional & 5 & 1.6 & 0 & 0 & $0.347(0.073-1.647)$ \\
\hline Others & 2 & 0.3 & 0 & 0 & $0.347(0.043-2.794)$ \\
\hline \multicolumn{6}{|l|}{ Residence } \\
\hline Urban & 184 & 27.6 & 15 & 66.3 & 1 \\
\hline Rural & 483 & 72.4 & 8 & 33.7 & $0.152^{* *}(0.064-0.385)$ \\
\hline \multicolumn{6}{|l|}{ Current Marital Status } \\
\hline Never married & 4 & 0.5 & 0 & 0 & 1 \\
\hline Married & 618 & 92.9 & 21 & 91.6 & $1.667(0.552-5.324)$ \\
\hline Living together & 10 & & 0 & 0 & $1(0,287-3.479$ \\
\hline Widowed & 9 & 1.4 & 0 & 0 & $1(0.249-4.024)$ \\
\hline Divorced & 13 & 2 & 0 & 0 & $1(0.285-3.503)$ \\
\hline Not living together & 3 & 0.4 & 2 & 8.4 & $346.5(0.185-648693.7)$ \\
\hline
\end{tabular}

${ }^{* *}$ Significant association $(\mathrm{P}<0.005)$

Those women who had ever a terminated pregnancy were 3.918(CI: 1.585-9.684) times likely to use LARCs when compared to who did not ever had a terminated pregnancy. The odds of using LARCs were 3.507(CI: 1.318-9.334) for those subjects who heard FP on radio than who did not and 3.365(CI: 1.544-7.336) for those who heard FP on television compared to those who did not. The odds of using LARCS were 5.398(CI: 1.204-24.195) higher in women who were told about other FP by health or FP worker than who did not told about. If the decision maker on using a method is mainly partner when compared with if it is mainly by the respondent, the odds of using LARCs were 0.0231(0.085-0.654) times higher in the former group.

Table 2. Percentage of Current Contraceptive Method Users; and Unadjusted Odds Ratios and 95\% Confidence Intervals for Current Use of Long-Acting Reversible Methods by Selected Reproductive Characteristics, Ethiopia, 2005.

\begin{tabular}{|c|c|c|c|c|c|}
\hline \multirow{3}{*}{$\begin{array}{l}\text { Reproductive characteristics } \\
\text { Ever had terminated pregnancy }(n=690)\end{array}$} & \multicolumn{4}{|c|}{ Method use } & \multirow{3}{*}{ Unadjusted OR (95\% CI) } \\
\hline & \multicolumn{2}{|c|}{ SARCS } & \multicolumn{2}{|c|}{ LARCS } & \\
\hline & NO & $\%$ & No. & $\%$ & \\
\hline No & 596 & 89.4 & 18 & 76 & 1 \\
\hline Yes & 70 & 10.6 & 5 & 24 & $3.918^{* *}(1.585-9.684)$ \\
\hline \multicolumn{6}{|l|}{ Number of living children $(\mathrm{N}=690)$} \\
\hline $1-5$ & 439 & 65.8 & 12 & 53.2 & 1 \\
\hline 6-11 & 228 & 34.2 & 11 & 46.8 & $1.742(0.658-4.611)$ \\
\hline \multicolumn{6}{|l|}{ Heard Family planning of radio $(\mathrm{N}=690)$} \\
\hline No & 395 & 59.2 & 7 & 28.6 & 1 \\
\hline Yes & 272 & 40.8 & 18 & 71.4 & $3.507^{* *}(1.318-9.334)$ \\
\hline \multicolumn{6}{|l|}{ Heard Family planning of TV(N=690) } \\
\hline No & 573 & 85.9 & 17 & 70.7 & 1 \\
\hline Yes & 94 & 14.1 & 6 & 29.3 & $3.365^{* *}(1.544-7.336)$ \\
\hline \multicolumn{6}{|c|}{ Told about other FP method by health or FP worker(N=434) } \\
\hline No & 389 & 90.7 & 3 & 61.4 & 1 \\
\hline Yes & 40 & 72.4 & 2 & 38.6 & $5.398^{* *}(1.204-24.195)$ \\
\hline \multicolumn{6}{|l|}{ Decision maker on using contraception $(\mathrm{N}=626)$} \\
\hline Mainly respondent & 125 & 20.6 & 6 & 29.2 & 1 \\
\hline Mainly husband, partner & 32 & 5.2 & 0 & 0 & $0.236(0.085-0.654)$ \\
\hline Joint decision & 448 & 74.2 & 15 & 70.8 & $0.635(0.215-1.874)$ \\
\hline
\end{tabular}

\footnotetext{
${ }^{* *}$ Significant association $(\mathrm{P}<0.005)$
}

After adjusting for socio-demographic and sexual and reproductive health variables which showed significant association in bivariate analysis, highest level of education attained, ethnicity, ever had terminated pregnancy, heard family planning on radio, told about FP method by health or FP worker and decision maker on contraception continued to have significant association for the use of LARCs in the study subjects.

The odds of using LARCS for those women who attained higher education were found to be $0.013(95 \%$ CI: 0.001-0.317) when compared to women with no education. Being from Hadiya ethnic group is 818 times more likely to use LARCs 
when compared with Amhara ethnic group (OR=818.012; 95\% CI 12.228-54720.501). Those women who had ever terminated pregnancy were 44433.258 times more likely to use LARCs (95\% CI: 6099.902-323663.1). The odds of using LARCs were 0.012(95\% CI: 0.001-0.284) in those subjects who heard about FP on radio last month. Women who were told about FP method by health or FP worker were $0.05(95 \% \mathrm{CI} /$; 0.007-.0377) times more likely to use LARCS and lastly if the decision maker on using contraception is mainly husband, the women is $19787.167(95 \% \mathrm{CI}$; 1415.5-276603.266) times more likely to use LARCs when compared to if the decision maker was mainly the respondent.

Table 3. Adjusted Odds Ratios and 95\% Confidence Intervals for Selected Respondent Characteristics and Current Long-Acting Reversible Contraception Use, Ethiopia, 2005.

\begin{tabular}{|c|c|c|}
\hline Socio-demographic characteristics & Adjusted OR & $95 \% \mathrm{CI}$ \\
\hline \multicolumn{3}{|l|}{ Education } \\
\hline No Education & 1 & - \\
\hline Primary & 1.345 & $0.120-15.074$ \\
\hline Secondary & 12.202 & $0.732-203.357$ \\
\hline Higher & 0.013 & $0.001-0.317^{* * *}$ \\
\hline \multicolumn{3}{|l|}{ Ethnicity } \\
\hline Amhara & 1 & \\
\hline Hadiya & 818.012 & $12.228-54720.501^{* * *}$ \\
\hline Harari & 1.169 & $0.045-30.625$ \\
\hline Oromo & 1.169 & $0.045-30.625$ \\
\hline Tigre & 129786.545 & $4747.127-3548366.4^{*}$ \\
\hline Others & 0.031 & $0.003-1.37$ \\
\hline \multicolumn{3}{|l|}{ Residence } \\
\hline Urban & 1 & - \\
\hline \multicolumn{3}{|l|}{ Ever had terminated pregnancy } \\
\hline No & 1 & \\
\hline Yes & 44433.258 & $6099.902-323663.1 * * *$ \\
\hline \multicolumn{3}{|c|}{ Heard Family planning on radio last month } \\
\hline No & 1 & \\
\hline Yes & 0.012 & $0.001-0.284 * * *$ \\
\hline \multicolumn{3}{|l|}{ Heard Family planning on TV last month } \\
\hline No & 1 & \\
\hline Yes & 0.781 & $0.054-11.317$ \\
\hline \multicolumn{3}{|c|}{ Told about other FP method by health or FP worker } \\
\hline No & 1 & \\
\hline Yes & 0.05 & $0.007-0.337 * * *$ \\
\hline \multicolumn{3}{|l|}{ Decision maker on using contraception } \\
\hline Mainly respondent & 1 & \\
\hline Mainly husband, partner & 19787.167 & $1415.5-276603.266 * * *$ \\
\hline Joint decision & 0.962 & $0.123-7.275$ \\
\hline
\end{tabular}

*** Significant association $(\mathrm{p}<0.05)$

\section{Discussion}

Prevention of unintended pregnancy requires appropriate use of contraceptive methods and women use contraception is either for limiting or postponing pregnancy for a certain period of time. Mothers who have no desire for extra children use different method of contraception to achieve their desire. This study aimed to identify Socio-demographic and sexual and reproductive factors affecting the use of LARCs among mothers who reported to had no desire for more children in the 2005 EDHS.

The use of LARCs was found to be significantly associated with education level; women who attained higher education were found to use the method than who have no education and the association was significant in binary analysis as well as after adjustment for other factors. This finding is consistent with other related studies [11, 22, 33]. The more the women is educated, the easier to understand messages on family planning choice and more importantly to make decision by comparing the long and short term benefit of any method.

Women who had ever terminated pregnancy were found to be significantly associated with the use of LARCs. This finding was contrary to one other study`s finding in which history of past abortion has nothing to do with the use of LARCS [22]. The study which was conducted to determine characteristics of women who are using LARCS in USA, focused on all women unlike the focus of this specific study which is women who did not have desire for more children. A women who experienced abortion but who did not decide to limit child bearing may not choose LARCs. But once a woman decided to limit the number of children, she needs a much more effective method if she experienced unwanted pregnancy due to past method failure and ended up in terminating it; and this association which remained significant in binary as well as after adjusting for other factors can be explained by the stated fact. 
Information about available contraceptive methods and the benefit of each method through mass media can contribute a lot for contraceptive use and informed method choice. This study was able to show that women who heard $\mathrm{FP}$ on radio and TV were more likely to use LARCs in the bivariate analysis. A study conducted in Kenya demonstrated similar finding and explained as those women who exposed to information through radio remained significant users of the methods after adjusting for other factors [9].

Mothers may visit health facilities for different reasons and personnel working at the facilities can play greate role in advocating for all family planning methods according to the need of individual women. This was backed up by an intervention done in Zambia to increase uptake of LARCS thorough dedicated midwifes in which it was able to provide this method for significant number of clients [23]. Even if the study did not revealed any significant association in those mothers whom at health facility told about FP unlike some studies [22], it was able to find a significant association for the use of LARCs methods among women who were told about FP methods by health or FP worker. It is not unlikely that health professionals working at health facilities were not giving sufficient information on the range of available contraceptive methods so that mothers can have contraceptive choice. This can be due to the time and procedure the LARCs need to explain [7] and insert apart from availability of the methods, the necessary equipment and appropriate infrastructure.

If the decision maker on use of contraception is mainly husband, the respondent is more likely to use LARCs and this was significant in the binary analysis and even after adjusting for other factors. This study did not address the factors which may influence the use of LARCs from the husband or partner characteristics however; but the finding could be attributed to a well informed husband or partner about available methods and their benefit.

One of the strength of this study is that it is based on a nationally representative data and thus it can be used as a reference for further studies. As it is secondary date all the limitations of secondary data applies for this study in addition to limitations peculiar to this study among which it did not take any factor from the husband/partner side.

\section{Conclusion and Recommendation}

This study has identified highest level of education attained, ever had a terminated pregnancy, Heard FP on radio, residence and decision maker on using contraception were found to be major determinates for the use of long acting reversible contraceptive method among women who desired no more children in Ethiopia based on the 2005 EDHS data. Some of the selected factors were not found to be determinant though has significant association and other selected variables failed to reveal any association.

One interesting finding of this study is that if the decision maker on the use of contraception is the husband or partner, the wife is highly likely to use LARCs as a method to limit the number of children. Capitalizing and addressing male partners on FP planning education and promotion means a lot to increase the uptake of a wide range of contraceptive methods and specifically on the long acting reversible ones. Mass media especially radio was also one area that could help to address the unmet need of family planning in the country and more specifically the advantageous LARCs.

Policy makers which work in the area of health and specifically on reproductive health area should give due attention to contraceptive utilization. They may indicate, in their policy document, that specific segment of population should use specific type of contraceptive methods based on the method specific benefit. Following introduction of policy programmers and implementers should focus on maximizing the utilization of LARCs in group of women who want to limit the number of children by using health talks and promotion how this method benefit these mothers. Finally researchers should dig out the best way to increase the utilization of the methods by continuously assessing the prevalence and factors which hinder or promote the use of these specific methods.

Recently the 2011 Ethiopian Demographic Health Survey has shown that there is a substantial increase in the usage of implants and a decrease in the use of pills among all women studied. In depth analysis of the factors that increased the use of LARCs and comparing with this study finding will give a clearer picture where to act to maximize the use of this specific segment of contraceptive options.

\section{Acknowledgements}

First and for most I would like to thank Dr. Alemayehu Worku for his guidance and professional impute starting from the development of thesis proposal through all the analysis part to the end of the journey. My gratitude also extends to Professor Yemane Berhane for his assistance on how I should proceed on my research project due to my relocation from Ethiopia. I would also like to thank Mrs. Seada Beyan for letting me know all the information from the university about schedules and other areas on time.

My gratitude also goes to ICF Macro for providing with the data set for analysis.

Lastly I appreciate the university of Gondar and Addis Continental Institute of Public health for giving me this opportunity and go one step in my career.

\section{Abbreviation}

CI-Confidence interval

DHS-Demographic Health Survey

EDHS-Ethiopian Demographic Health Survey

FMOH-Federal Ministry of Health

FP-Family Planning

IUCD-Intrauterine contraceptive Device

IUD-Intrauterine Device 
LARCs-Long Acting Reversible Contraceptives

OCPs-Oral Contraceptive Pills

OR-Odds Ratio

RH-Reproductive Health

SARCs-Short Acting Reversible Contraceptive Methods

SPSS-Statistical Package for Social Science

USA-United States of America

WHO-World Health Organization

\section{Assurance of Principal Investigator}

I the undersigned agree to accept all the responsibilities for the scientific and ethical conduct of the research project. I will provide timely progress report to my advisor and seek the necessary advice and approval from my primary advisors in the course of the research. I will communicate timely to my advisors all stakeholders involved in the study including any source of funding for this research.

Name of the student Seyfu Abebe Desta

Signature

Date September 19, 2011

Approval of the primary advisor

Name of the primary advisor Dr. Alemayehu Worku

Signature

Date

\section{Appendix}

Study tools

Please see the EDHS 2005

Consent form

Hello. My name is

and I am working with the Population and Housing Census Commission Office (PHCCO). We are conducting a national survey about the health of women, men and children. We would very much appreciate your participation in this survey. I would like to ask you about your health (and the health of your children). This information will help the government to plan health services. The survey usually takes about 45 minutes to complete.

Whatever information you provide will be kept strictly confidential and will not be shown to other persons.

At this time, do you want to ask me anything about the survey?

May I begin the interview now?

Declaration

I, the undersigned declare that this thesis is my original work in partial fulfillment of the requirement for the degree of Master of Public Health. I also declare that it has never been presented in this or any other university and that all resources and materials used in the thesis have been duly acknowledged.

Student Name: Seyfu Abebe Desta

Signature:

Place of submission: Addis Ababa, Ethiopia

Date of submission: April, 2012

This thesis has been submitted for examination with my approval as a university advisor.

Advisor Name: Dr. Alemayehu Worku

Signature:

Date of submission:

\section{References}

[1] Hubacher D, Mavranezouli I, McGinn E. Unintended Pregnancy in Sub-Saharan Africa: Magnitude of the Problem and Potential Role of Contraceptive Implants. Contraception 2008; 78:73-78.

[2] United nations, Department of Economic and Social Affairs, Population Division (2011). World Contraceptive Use 2010(POP/DB/ Rev 2010).

[3] Ethiopia Central Statistical Authority. Ethiopian Demographic Health Survey 2000.

[4] Ethiopia Central Statistical Authority. Ethiopian Demographic Health Survey 2005.

[5] Osei I, Birungi H, Addico G, Askew I, Gyapong JO. What Happened to the IUD in Ghana? African Journal of Reprod Health 2005:9(2):76-95.

[6] McDonald-Mosley R., Philips K., Ditzian L., Cremer M. Acceptability of the Intrauterine Device among Women in El Salvador. International Journal of Gynecology And Obstetrics 2010; 108:152-160.

[7] Katz KR., Johnson LM., Janowitz B, Carranza JM. Reason for the Low Level of IUD Use in El Salvador. International Family planning Perspective 2002; 28(1):26-31.

[8] Thang NM, Huong VT. Changes In Contraceptive Use in Vietnam. J Biosoc sci. 2003; 35:527-543.

[9] Magadi MA, Curtis SL. Trends and Determinant of Contraceptive Method Choice in Kenya. Studies in family planning 2003; 34(3):149-159.

[10] Seiber EE, Bertrand JT, Sullivan TM. Changes in Contraceptive Method Mix In Developing Countries. International Family Planning Perspective 2007; 33(3):117-123.

[11] Brown W, Ottney A, Nguyen S. Breaking the Barrier: The Health Belief Model and Patient Perceptions Regarding Contraception. Contraception 2011:83(5):453-458.

[12] Steiner MJ., Trussell J., Mehta N., Condon S., Subramaniam S., Bourne D. Communicating contraceptive effectiveness: A randomized controlled trial to inform a World Health Organization family planning handbook. American Journal of Obstetrics and Gynecology 2006; 195: 85-91.

[13] Trussell J. Contraceptive failure in the United States. Contraception 2004; 70:89-96.

[14] Meirik O, Farley TM, Sivin I. Safety and efficacy of Levnogestril Implants, Intrauterine Device, and sterilization. Obst Gynecol 2001; 97:539-471.

[15] Clare Lipetz C., Phillips CJ., Fleming CF. The cost-effectiveness of a long-acting reversible contraceptive (Implanon ${ }^{\circledR}$ ) relative to oral contraception in a community setting. Contraception 2009; 79:304-309. 
[16] Mavranezouli I, Wilkinson C. Long-acting reversible contraceptives: not only effective, but also a cost-effective option for the National Health Service. J Fam Plann Reprod Health Care. 2006; 32(1):3-5.

[17] Frost JJ, Darroch JE. Factors Associated with contraceptive choice and inconsistent method use, United States. 2004. Perspectives on Sexual and Reproductive Health, 2008, 40(2):94-104, doi: 10.1363/4009408.

[18] Kane R, Irving G, Brown S, Parkes N, Walling M, Killick S. Long acting, reversible and permanent method of contraception; Insight in to women's choice of method. Quality in primary care 2009; 17:107-114.

[19] Mitchel MJ, Thistle P. Acceptability of levonorgestrel subdermal implants versus tubal ligation for long-term contraception in a rural population of Zimbabwe Contraception 2004; 70(6):483-486.

[20] Baveja R, Buckshee K, Das K, Das SK, Hazra MN, Gopalan S Evaluating contraceptive method choice using the method mix approach. Contraception 2000; 61:113-119.

[21] Renee C., Wong RC., Bell RJ, Thunuguntla K., McNamee K., Vollenhovena B. Implanon users are less likely to be satisfied with their contraception after 6 months than IUD users Contraception 2009; 80:452-456.

[22] Kavanaugh ML, Jerman J, Hubacher D, Kost K, Lawrence B. Finer LB. Characteristics of women in the US who use Long acting reversible methods. Obstet Gyncol 2011; 117:1349-57

[23] Neukoma J, Chilambweb J, Mkandawireb J, Mbewec RK, Hubacherd D. Dedicated providers for long acting reversible contraception: new approach in Zambia. Contraception 2011; $83: 447-452$.

[24] Gutin SA., Mlobeli R, Moss M, Buga G, Morroni C. Survey of knowledge, attitude and practice surrounding the intrauterine device in South Africa. Contraception 2011; $83: 145-150$

[25] Ali M, Cleland J. Determinant Of Contraceptive Discontinuation In Six Developing Countries. J. Biosoc, sci 1999; 31:343-360.

[26] Gilliam ML, Warden M., Goldstein C., Tapia B. Concerns about contraceptive side effects among young Latinas; a focus group approach. Contraception 2004; 70:299-305.

[27] Fertility and family planning in Ethiopia, A new look at data from the 2005 Ethiopia Demographic and Health survey.

[28] Ruminjo JK., Amatya RN., Dunson TR., Krueger SL., Chi I. Norplant implants, Acceptability and User Satisfaction Among Women in Two African Countries. Contraception 1996; 53:101-107.

[29] Rivera R., Chen-Mok M., McMullen S. Analysis of client characteristics that may affect early discontinuation of copper IUD. Contraception 1999; 60:155-60.

[30] Hubacher D., Goco N., Gonzalez B, Doug Taylor D. Factors affecting continuation rate of DMPA. Contraception 2000; 60:345-351.

[31] Summary and Statistical Report of the 2007 Population and Housing Census. Federal Democratic Republic of Ethiopia Population census commission; December 2008.

[32] Hubacher D, Kimani J, Steiner MJ, Solomon M, Ndugga MB. Contraceptive Implants in Kenya; current practice and future prospects. Contraception 2007; 75:468-473.

[33] Alemayehu M. Belachew T., Tizta T. Factors associated with the utilization of long and permanent contraceptive methods among married women of reproductive age in Mekelle town, Tigray region, north Ethiopia. BMC Pregnancy and Childbirth 2012 12:6. 